\title{
Effect of Additional Cross-linking of Continuous Chemical Stress Relaxation of cis-Polybutadiene
}

\author{
Arthur V. Tobolsky, Yuzo TAKahashi, ${ }^{*}$ and Shunji Naganuma* \\ Department of Chemistry, Princeton University, Princeton, \\ New Jersey 08540, U.S.A.
}

(Received July 6, 1971)

\begin{abstract}
Continuous and intermittent stress relaxation experiments were carried out on dicumyl peroxide cured cis-polybutadiene in high vacuum and in air at several temperatures. An extension of the "two-network theory" is proposed whereby the effectiveness of a cross-link at various extension ratios is experimentally defined. The effectiveness varies from unity at an extension ratio of unity to zero at very high extension ratios.
\end{abstract}

KEY WORDS cis-Polybutadiene / Continuous Stress Relaxation / Cross-linking in Stretched State / Vacuum / Stress Increase / Effective Cross-linking / Effectiveness /

It is an interesting and important problem whether the new cross-linkage contributes to the observed force in a sample held at constant strain. A number of investigations, ${ }^{1-7}$ both theoretical and experimental, have been carried out on this subject. These investigations give conflicting conclusions about the effect of additional cross-linkings; some of them support the repression of the stress decay and others indicate no repression exists.

In this work, the techniques of continuous stress relaxation and intermittent stress relaxation measurements ${ }^{8}$ were used to measure separately the effects of scission and cross-linking of polymers. Under continuous stress relaxation measurements of cis-polybutadiene in vacuo, an obvious stress increase without shrinkage of the sample was observed but this did not occur in air.

Our experimental findings suggest that the "two-network theory," which predicts no contribution of the new cross-linkages to the observed force, needs to be improved and extended.

\section{EXPERIMENTAL}

\section{Materials}

The samples were cured cis-polybutadiene

* The Central Research Laboratory of Hokkai Seikan Co., 839-1, Kanamuro, Iwatsuki, Saitama, Japan.
Table I. Composition and curing condition of samples

\begin{tabular}{lccc}
\hline Component & $\begin{array}{c}\text { Budene } \\
501\end{array}$ & $\begin{array}{c}\text { Dicumyl } \\
\text { peroxide }\end{array}$ & $\begin{array}{c}\text { Curing } \\
\text { condition }\end{array}$ \\
\hline Sample A & 100 & 2.0 & $40 \mathrm{~min}$ at $135^{\circ} \mathrm{C}$ \\
Sample B & 100 & 0.6 & $90 \mathrm{~min}$ at $135^{\circ} \mathrm{C}$ \\
\hline
\end{tabular}

(Budene 501) supplied by the Goodyear Tire and Rubber Company. The particulars are shown in Table I.

All samples used here were subjected to thorough Soxhlet extraction in cyclohexane and acetone at their boiling temperatures for $48 \mathrm{hr}^{10}$ respectively after vulcanization, to remove chemical residues that might act as cross-linking agents. Ninety minutes was the minimum acceptable curing time for sample $\mathbf{B}$; with shorter time curing the samples produced were appreciably soluble in cyclohexane, benzene, and so on.

\section{Equipment and Procedure}

Stress relaxation measurements were conducted both in air and in vacuo $(0.001 \mathrm{mmHg})$ using a spring relaxometer. ${ }^{11,12}$ The sample used for in vacuo measurements were conditioned in vacuo at $120^{\circ} \mathrm{C}$ for $16 \mathrm{hr}$ before each run to eliminate oxygen molecules in the sample. In both air and vacuum measurements, the temperature was raised rapidly to the test temperature and held one hour. The sample was then extended 
and force $v_{s}$. time measurements were started.

The number of network chains was calculated with the stress $F(t)$ at time $t$ by the following equation.

$$
F(t)=2 C(t) R T\left(\alpha^{2}-\frac{1}{\alpha}\right)
$$

where $2 C(t)$ is the number of network chains $(\mathrm{mol} / \mathrm{cc})$ at time $t, \alpha$ is an extension ratio, $R$ is gas constant, and $T$ is temperature $\left({ }^{\circ} \mathrm{K}\right)$.

The initial number of network chains $2 C(0)$ of samples $\mathrm{A}$ and $\mathrm{B}$ at $230^{\circ} \mathrm{C}$ in vacuo were about $2.8-3.0 \times 10^{-5} \mathrm{~mol} / \mathrm{cc}$ and $2.4-2.6 \times 10^{-4}$ $\mathrm{mol} / \mathrm{cc}$ respectively.

\section{RESULTS AND DISCUSSION}

Figure 1 shows continuous stress relaxation curves relative stress $F_{\mathrm{c}}(t) / F_{\mathrm{c}}(0)$ vs. time of sample $A$ in vacuo over the temperature range 200 to $260^{\circ} \mathrm{C}$. The observed stress decay is slower than expected from some previous reports. After the relative stress becomes about 0.85 to 0.90 , the stress increases with temperature. After each run of measurements, the length of the sample was checked returning to zero stress and no shrinkage could be observed within experimental errors (within $0.1 \%$, about $0.1 \mathrm{~mm}$ ). Figure 2 shows results of sample $A$ in air. No stress increase was observed. Figure 3 shows results of continuous and intermittent stress relaxation measurements. The figure also includes results of SMCIR (simultaneous measurements of continuous and intermittent stress relaxation $)^{13}$ for sample $\mathrm{A}$ in vacuo at $230^{\circ} \mathrm{C}$. In this case, the first extension ratio $\alpha_{1}$ was 1.01 and the second one $\alpha_{2}$ was 1.015. The stress increases steadily in intermittent measurements. In Figure 3, the stress increasing curve for intermittent measurements is in a good agreement with that for intermittent of SMCIR at $\alpha_{1}=1.01$. It is known that the stress increase in intermittent measurements is caused by cross-linking. The good agreement of the results in two intermittent measurements means that cross-linking reaction does not depend on whether the sample is stretched or not. Figure 3 also shows that the stress in continuous measurements increases after a certain period and that these curves approach that in intermittent measurements with decreasing $\alpha$. These facts show that the stress increase is caused by cross-

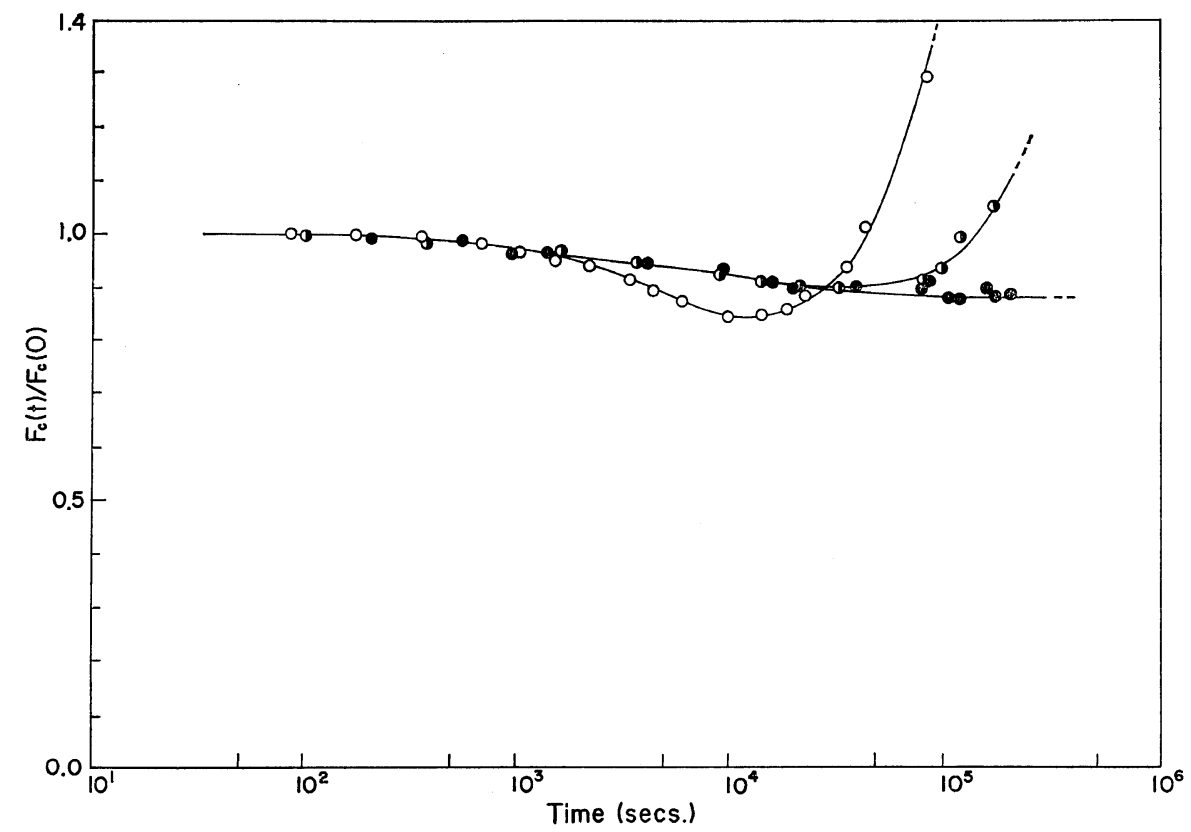

Figure 1. Continuous stress relaxation curves of sample $\mathrm{A}$ in vacuo at $200^{\circ} \mathrm{C}(0, \alpha=1.050)$, $230^{\circ} \mathrm{C}(\mathcal{}, \alpha=1.050)$ and $260^{\circ} \mathrm{C}(\bigcirc, \alpha=1.050)$. 
A. V. Tobolsky, Y. Takahashi, S. Naganuma

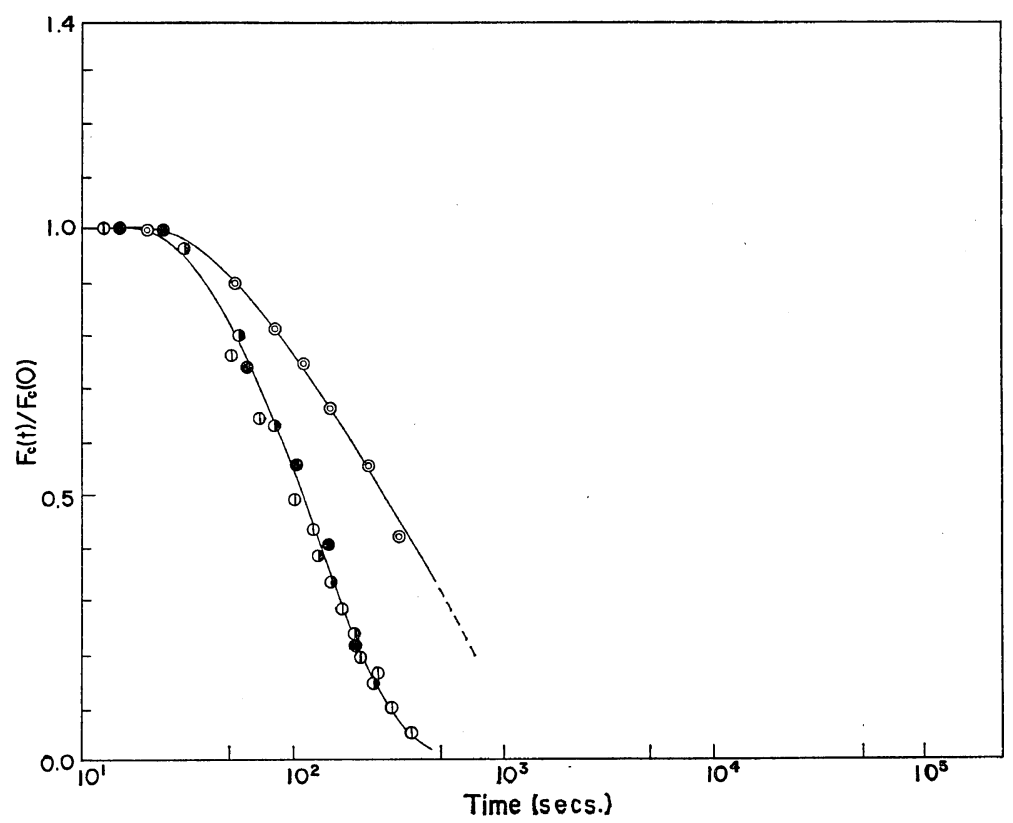

Figure 2. Continuous stress relaxation curves of sample $\mathrm{A}$ in air at $200^{\circ} \mathrm{C}(\bigcirc, \alpha=1.050$ ) and $\left.230^{\circ} \mathrm{C}(1), \alpha=1.010 ; \bigcirc, \alpha=1.050 ; 0, \alpha=1.050\right)$.

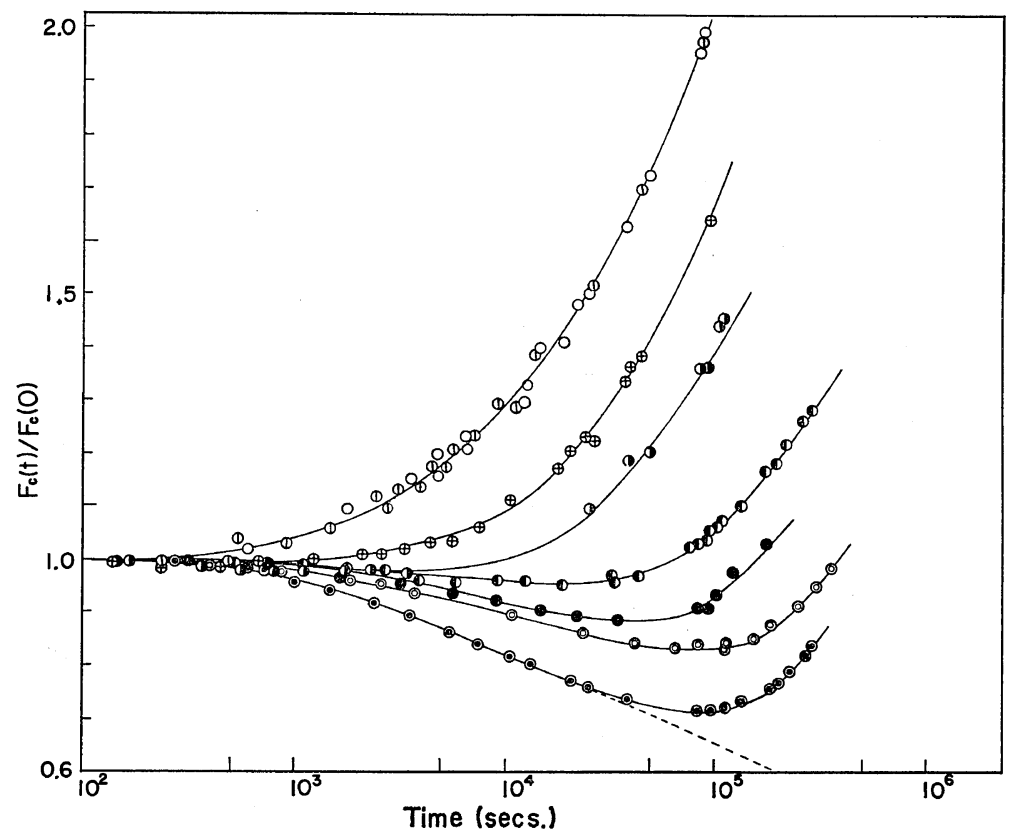

Figure 3. Intermittent stress relaxation curves ( $\bigcirc$, by usual method; (1), by SMCIR at $\alpha_{1}=$ 1.010) and continuous stress relaxation curves at various extension ratios $(\oplus, 1.005$; $($, 1.010 ; $1.1 .025 ; 0,1.050$; $\bigcirc, 1.100 ; \bigcirc, 1.133$ ) in vacuo at $230^{\circ} \mathrm{C}$ of sample $\mathrm{A}$. 


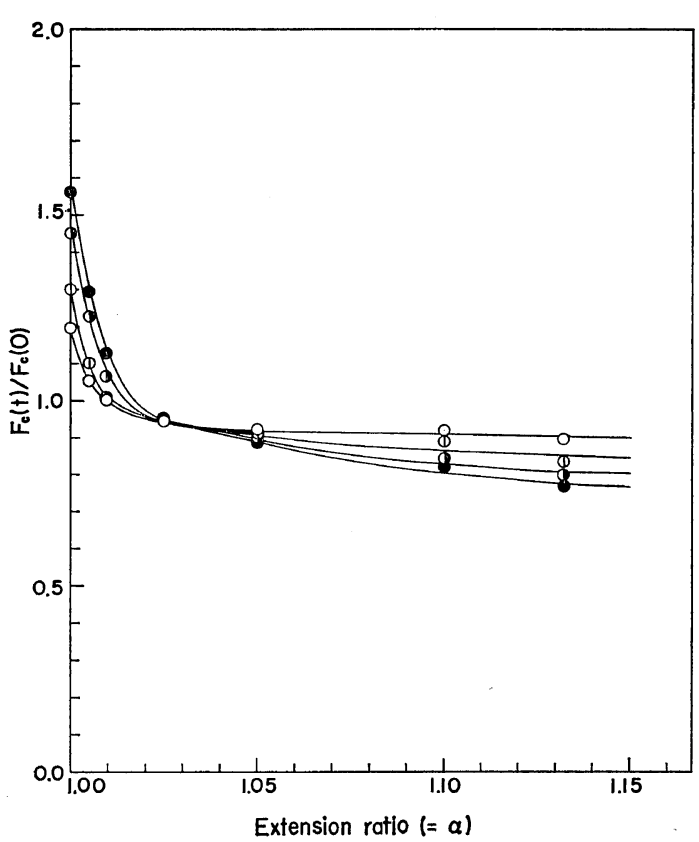

Figure 4. Extension ratio dependence of the relative stress for continuous stress relaxation measurements in vacuum at $230^{\circ} \mathrm{C}$ as a function of time: $\bigcirc, 500 \mathrm{sec}$; (1), $1000 \mathrm{sec}$; $2000 \mathrm{sec}$; $3000 \mathrm{sec}$. linking even in the stretched sample and that the contribution of cross-linking to the stress depends on $\alpha$. Figure 4 shows plots of relative stress at arbitrary times in Figure 3 against $\alpha$. The relative stress seems to level off at $\alpha$ larger than 1.05. The smaller increase of the relative stress for the larger $\alpha$ in Figure 3 and the behavior of levelling off in the relative stress in Figure 4 suggests that the stress increase in continuous stress relaxation measurements disappears for large $\alpha$. The dotted curve in Figure 3 is drawn assuming that the stress increase disappears. We denote this relative stress as $\left[F_{\mathrm{c}}(t) / F_{\mathrm{c}}(0)\right]_{\infty}$ upon considering the concept of "two-network theory."

The magnitude of the stress increase at an arbitrary extension ratio $\alpha$ can be given by the difference in the relative stress at $\alpha,\left[F_{\mathrm{c}}(t) /\right.$ $\left.F_{\mathrm{c}}(0)\right]_{\alpha}$, and $\left[F_{\mathrm{c}}(t) / F_{\mathrm{c}}(0)\right]_{\infty}$. The value of $\left[F_{\mathrm{c}}(t) /\right.$ $\left.F_{\mathrm{c}}(0)\right]_{\alpha}-\left[F_{\mathrm{c}}(t) / F_{\mathrm{c}}(0)\right]_{\infty}$ for sample $\mathrm{A}$ in vacuo at $230^{\circ} \mathrm{C}$ are shown as functions of time at each extension ratio in Figure 5. If we multiply a suitable value $1 / \beta$ to each curve in Figure 5 , we can superpose all of the curves on the intermittent curve as shown in Figure 6. The factor $1 / \beta$ depends on $\alpha$ but not on time. In this

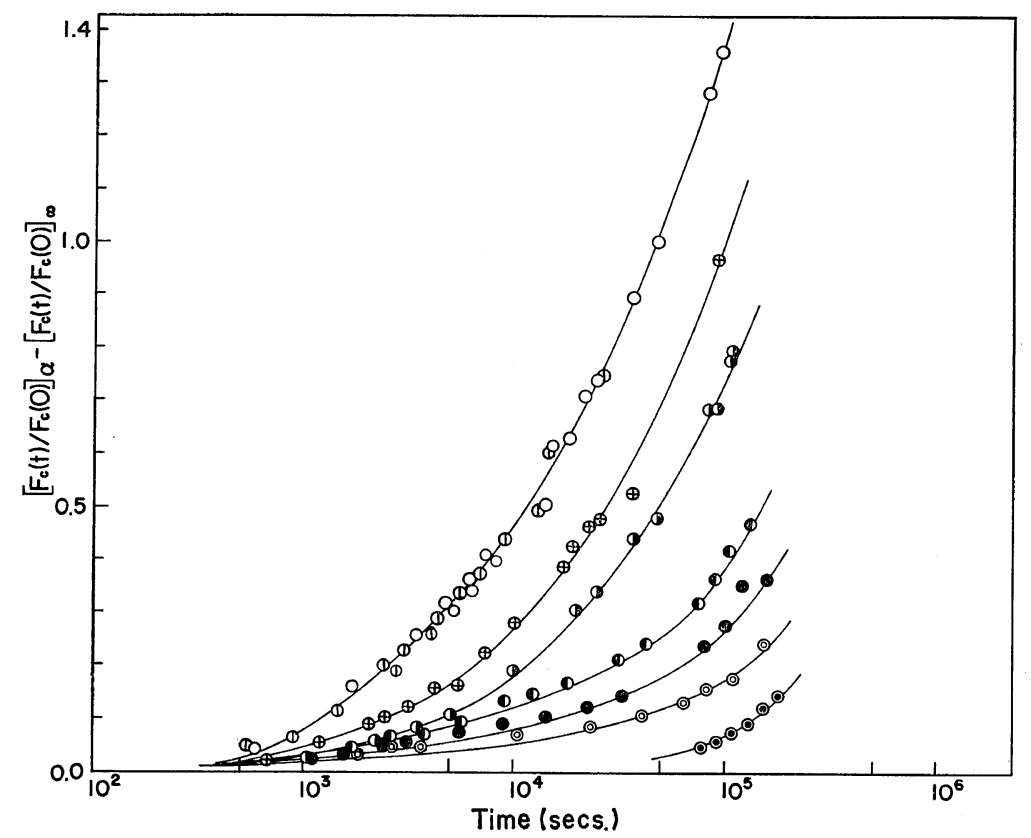

Figure 5. $\left\{\left[F_{\mathrm{c}}(t) / F_{\mathrm{c}}(0)\right]_{\alpha}-\left[F_{\mathrm{c}}(t) / F_{\mathrm{c}}(0)\right]_{\infty}\right\}$ curves of sample $\mathrm{A}$ obtained from Figure 3 as a function of extension ratios. Each symbol is the same as in Figure 3. 


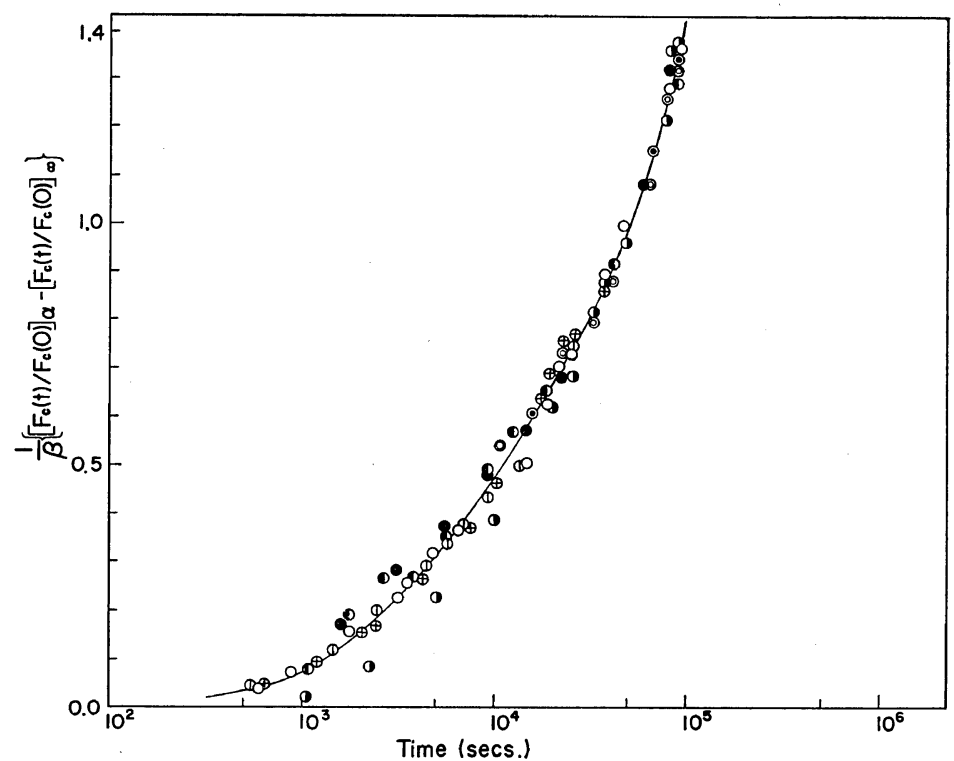

Figure 6. Superposed curves of $1 / \beta\left\{\left[F_{\mathrm{c}}(t) / F_{\mathrm{c}}(0)\right]_{\alpha}-\left[F_{\mathrm{c}}(t) / F_{\mathrm{c}}(0)\right]_{\infty}\right\}$ of sample $\mathrm{A}$ in vacuo at $230^{\circ} \mathrm{C}$. Each symbol is the same as in Figure 3.

procedure, $\beta$ is expressed by the following as shown in Figure 8 . equation

$$
\beta=\frac{\left[F_{\mathrm{c}}(t) / F_{\mathrm{c}}(0)\right]_{\alpha}-\left[F_{\mathrm{c}}(t) / F_{\mathrm{c}}(0)\right]_{\infty}}{\left[F_{1}(t) / F_{\mathrm{i}}(0)\right]-\left[F_{\mathrm{c}}(t) / F_{\mathrm{c}}(0)\right]_{\infty}}
$$

where suffixes $\mathrm{c}$ and $\mathrm{i}$ of $F$ mean continuous and intermittent stress relaxation respectively.

The denominator of eq 2 shows the contribution of cross-linking to the stress increase at zero extension and the numerator is that at an extension of $\alpha$. Therefore, $\beta$ is considered as a fraction of the effective contribution of cross-linkings to the stress increase at $\alpha$. We call it "effectiveness."

Plots of the effectiveness $\beta$ vs. $\alpha$ are shown in Figure 7. Here, we use the relative stress at $\alpha=1.133$ in place of $\left[F_{\mathrm{c}}(t) / F_{\mathrm{c}}(0)\right]_{\infty}$ according to the above discussions because $\alpha=1.133$ is the maximum extension ratio without breakage of samples in this work. The effectiveness rapidly decreases in the region of small $\alpha$ and slowly approaches zero for large $\alpha$. The $\alpha$ dependence of the effectiveness $\beta$ becomes smaller above $\alpha=1.10$. This fact also supports the use of the relative stress at $\alpha=1.133$ in place of $\left[F_{\mathrm{c}}(t) / F_{\mathrm{c}}(0)\right]_{\infty}$.

Cross-linking formations in the stretched sample are classified roughly into two groups (a) Cross-linking of a free chain in the network structure.

(b) Cross-linking of stretched network chains.

In the case of cross-linking (a), the chain BE is always in thermal equilibrium and not in extension. Therefore, cross-linking (a) contributes nothing to the stress in continuous stress relaxation measurements as the "two-network theory" indicates.

In the case of cross-linking (b), two network chains will be brought together. By cross-linking reactions, two network chains form four network chains. Each network chain will be extended to various extension ratios; some of them are larger than $\alpha$ and others smaller than $\alpha$. But the averaged extension ratio of these network chains in the direction of the stress may be equal to $\alpha$ on the basis of the network theory of rubber. This is the reason why the shrinkage of the sample could not be observed. The stress increase is explained by the increase in the number of network chains formed by cross-linking (b).

We denote the number of increased network chains formed by cross-linking (a) as $2 \Delta C_{\mathrm{a}}(t)$ and that by cross-linking (b) as $2 \Delta C_{\mathrm{b}}(t)$. Then, the stress in continuous stress relaxation meas- 


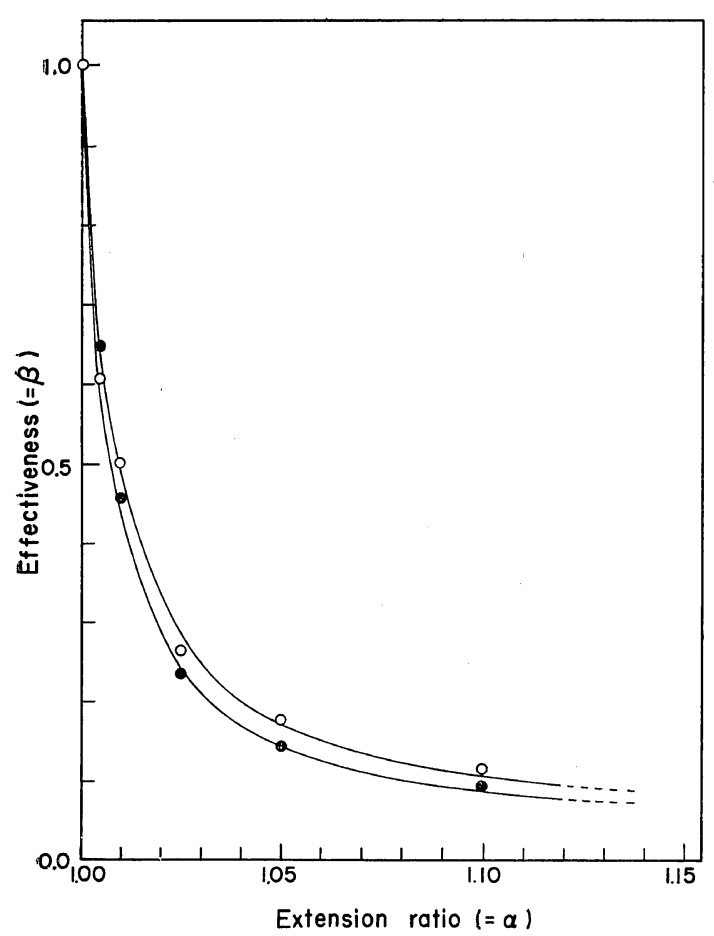

Figure 7. Effectiveness $\beta$ vs. extension ratio $\alpha$ of samples $\mathrm{A}$ and $\mathrm{B}$ in vacuo at $230^{\circ} \mathrm{C}$ : $\bigcirc$, sample A; $\mathbf{O}$, sample B.

(a) Crosslinking of a free chain

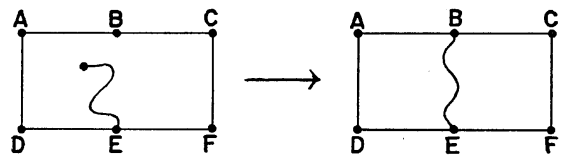

(b) Crosslinking of network chains

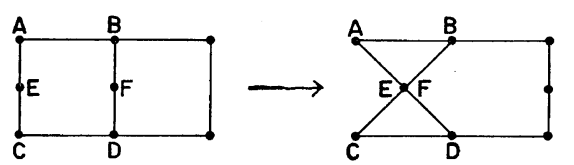

Figure 8. Classification of cross-linking reactions in network structures.

urements in which only cross-linking (b) contributes to the stress is written as follows

$$
F_{\mathrm{c}}(t)=\left[2 C(0)-q(t)+2 \Delta C_{\mathrm{b}}(t)\right] R T\left(\alpha^{2}-\frac{1}{\alpha}\right)
$$

where $2 C(0)$ is the initial number of network chains and $q(t)$ is the decreased number of network chains by scission reactions for time $t$, and the stress in intermittent stress relaxation measurements in which cross-linking (a) and (b) contribute to the stress increase is

$F_{\mathrm{i}}(t)=\left[2 C(0)-q(t)+2 \Delta C_{\mathrm{a}}(t)+2 \Delta C_{\mathrm{b}}(t)\right] R T\left(\alpha^{2}-\frac{1}{\alpha}\right)$

When the cross-linking does not contribute to the stress increase, the stress is

$$
F_{\mathrm{c}}(t)=[2 C(0)-q(t)] R T\left(\alpha^{2}-\frac{1}{\alpha}\right)
$$

Substituting eq 3 , eq 4 , and eq 5 into eq $2, \beta$ is given by eq 6

$$
\beta=\frac{\Delta C_{\mathrm{b}}(t)}{\Delta C_{\mathrm{a}}(t)+\Delta C_{\mathrm{b}}(t)}
$$

Equation 6 shows that the effectiveness $\beta$ indicates the fraction of network chains formed by cross-linking (b). $\beta$ does not show time dependence as seen in Figure 6 and this behavior is explained easily using eq 6 with the concepts of cross-linking reactions. Cross-linking reagents, dicumyl peroxide, react with double bonds to make cross-links. There will exist no difference between two double bonds, one on a free chain and another on a network chain. This means that cross-linking reactions (a) and (b) have the same mechanism and that the time dependence of both cross-linking reactions are the same. According to these considerations, it will be apparent that the time dependence of eq 6 can be cancelled out between the numerator and the denominator and, therefore, $\beta$ shows no time dependence. These facts also mean that the fraction of network chains formed by crosslinking (b) is controlled only by the extension ratio $\alpha$.

Using $\alpha$ dependence of $\beta$, it will be possible to analyse $\alpha$ dependence of the reactivity of functional groups and to control the number of the network chains which have a certain direction. This is an interesting and important problem.

$\beta$ is obtained by continuous stress relaxation measurements as seen previously. But in air, it is impossible to observe exactly the stress increase as shown in Figure 2 even if cross-link- 
ing (b) occurs, because the stress decays at high velocity owing to oxidative scissions. Moreover, in air, the velocity of the stress decay in continuous stress relaxation measurements should be restrained by the contribution to the stress by cross-linking (b). If continuous stress relaxation measurements are done in high vacuum, repressing oxidative stress decay, for the usual natural and synthetic rubbers, the stress increase will be observed in the region of small $\alpha$ same as cis-polybutadiene in this report.

P. J. Flory, et al. ${ }^{1-4}$ reported that cross-linking reactions repress the stress decays in continuous stress relaxation measurements but, in practice, not only the repression of the stress decay but also stress increase was seen. This means that their theory is adaptable only for systems in which scission reactions occur very actively or at large extension ratios and that they are not sufficient to explain the contribution to the stress by cross-linking reactions in the stretched sample.

Usually, continuous stress relaxation measurements are performed with large extension ratios, larger than 1.2. In such a case, the effectiveness $\beta$ is very small as presumed from Figure 7 and this means also that the stress increase becomes negligibly small. The "two-network theory," therefore, assumes an approximate validity.

\section{CONCLUSION}

Stress increase, in the absence of sample shrinkage, under continuous stress relaxation measurements of cis-polybutadiene was observed in vacuo, $(0.001 \mathrm{mmHg})$-but not in air-over a temperature range $200^{\circ}$ to $260^{\circ} \mathrm{C}$. In vacuo, cross-linking appears to predominate over scission.

It was infered from experimental results that cross-linkings in the stretched sample contribute to the stress to an extent depending on the extension ratio $\alpha$ and the concept of effective cross-linkings which contribute to the stress was introduced. To show the effective cross-linkings quantitatively, the term "effectiveness" was used-which is given as follows experimentally -and the relation between $\alpha$, cross-linking reactions and "effectiveness" was discussed.

$$
\text { Effectiveness }=\frac{\left[F_{\mathrm{c}}(t) / F_{\mathrm{c}}(0)\right]_{\alpha}-\left[F_{\mathrm{c}}(t) / F_{\mathrm{c}}(0)\right]_{\infty}}{\left[F_{\mathrm{i}}(t) / F_{\mathrm{i}}(0)\right]-\left[F_{\mathrm{c}}(t) / F_{\mathrm{c}}(0)\right]_{\infty}}
$$

The effectiveness is unity at $\alpha=1$, and according to the experimental results, may approach zero at $\alpha \rightarrow \infty$.

From this investigation, it has been found that the "two-network theory" is applicable at relatively large $\alpha$, even though the concept of "effectiveness" must be invoked.

Acknowledgement. The authors wish to thank Prof. M. Kaneko and Associate Prof. K. Hikichi of Hokkaido University and Prof. K. Murakami of Tohoku University for their interest and helpful advice.

\section{REFERENCES}

1. P. J. Flory, Trans. Faraday Soc., 56, 722 (1960).

2. J. Scanlan, Trans. Faraday Soc., 57, 839 (1961).

3. D. K. Thomas, Polymer, 7, 125 (1966).

4. J. Berry, W. F. Watson, and J. Scanlan, Trans. Faraday Soc., 52, 1137 (1956).

5. K. Murakami, et al., J. Soc. Materials Sci., Japan, 15, 312 (1966).

6. K. Murakami, T. Kusano, S. Naganuma, and Y. Takahashi, Bull. Chem. Research Institute of Non-Aqueous Solutions, Tohoku Univ., Japan, 19, 243 (1969).

7. S. Naganuma and Y. Takahashi, Kobunshi Kagaku (Chem. High Polymers, Japan), 27, 705 (1970).

8. A. V. Tobolsky, "Properties and Structure of Polymers," John Wiley and Sons, Inc., New York, N.Y., 1960, Chapter V.

9. R. D. Andrews, A. V. Tobolsky, and E. E. Hanson, J. Appl. Phys., 17, 352 (1946).

10. J. R. Dunn, J. Appl. Polym. Sci., 7, 1543 (1963).

11. M. T. Shaw and A. V. Tobolsky, ONR Technical Report, Task No. NR 056-377, Office of Naval Research, December, 1968, No. 111.

12. Y. Takahashi and A. V. Tobolsky, Polymer J., Japan, in press.

13. H. Sobue, K. Matsuzaki, T. Migita, and K. Murakami, Kobunshi Kagaku (Chem. High Polymers, Japan), 21, 606 (1964). 